\title{
El bajo consumo de alimentos ricos en hierro y potenciadores de su absorción se asocia con anemia en preescolares cubanos de las provincias orientales. 2005-2011
}

\section{Low consumption of iron-containing foods and enhancer of iron absorption are associated with anemia in preschool children of the eastern provinces of Cuba. 2005-2011}

\begin{abstract}
Anemia in children less than five years of age is the main nutritional problem in Cuba. The aim of this study was to assess the intake of iron-rich foods (IRF) and of enhancers of iron absorption (EIA) in children living in the five eastern provinces of Cuba and its association with anemia in 2005, 2008 and 2011. A food frequency questionnaire of 6 months and hemoglobin determination were used. Intake increased in red meat and poultry (44.2\% to $60.4 \%)$, eggs (38.7\% to $58.6 \%$ ) and vegetables $(47.6 \%$ to $56.2 \%)$; decreased in fruit $(73.2 \%$ to $55.8 \%)$ and legumes ( $87.7 \%$ to $57.7 \%)$. The prevalence of anemia in children 6 to 11 months old showed a decrease $(62.1 \%$ to $44.3 \%)$. A reduction in the prevalence of anemia in infants 12-23 months old was found in 2008 compared with 2005 and reversed slightly in 2011, a similar finding was observed in children 2-5 years old. There was a consistent association of anemia with low intake of eggs, vegetables and fruits, but and not with low intake of legumes. Children who attended daycare center ate more iron-rich foods and enhancers of iron absorption than those who did not. The group of children younger than 24 months old remains the most vulnerable group for anemia.

Key words: meals rich in iron, anemia; toddlers; daycare center.
\end{abstract}

\section{INTRODUCCIÓN}

La anemia en los niños menores de 5 años constituye el principal problema nutricional por deficiencia específica de hierro y se considera un problema de salud pública en Cuba (1). Las causas de la anemia pueden ser multifactoriales y a menudo coincidentes en el tiempo, pero la principal es la baja ingestión de alimentos con fuentes adecuadas de hierro en cantidad y calidad; la literatura expone que el 50\% de las causas de anemia resulta de la deficiencia de hierro (2). El porcentaje de absorción del hierro no hemínico depende del
Gisela Pita-Rodríguez (1) Santa Jiménez-Acosta (1) Beatriz Basabe-Tuero (1)

Consuelo Macías Matos (1)

Lisette Selva Suárez (2) Clarisa Hernández Fernández (2) Margarita Cruz Jorge (3)

Roxana Herrera Charro (4) Regla O'Farrill López (3) Isabel Calderius Espinosa (3) Karelia Paulí Echevería (5) Moraima Leyva Ávila (6)

(1) Depto Bioquímica y Fisiología. Instituto de Nutrición e Higiene de los Alimentos. La Habana, Cuba.

(2) Centro Provincial de Higiene y Epidemiología, Holguín, Cuba (3) Centro Provincial de Higiene y Epidemiología, Granma, Cuba (4) Centro Provincial de Higiene y Epidemiología, Guantánamo, Cuba (5) Centro Provincial de Higiene y Epidemiología, Santiago de Cuba, Cuba (6) Centro Provincial de Higiene y Epidemiología, Las Tunas, Cuba

$$
\begin{array}{r}
\text { Dirigir la correspondencia a: } \\
\text { Profesora } \\
\text { Gisela Pita-Rodríguez } \\
\text { Depto. Bioquímica y Fisiología. } \\
\text { Instituto de Nutrición e Higiene de los Alimentos. } \\
\text { Infanta 1158. Habana 10300. Cuba } \\
\text { Teléfono:0537-8795183 } \\
\text { E-mail: hematologia@sinha.sld.cu }
\end{array}
$$

Este trabajo fue recibido el 24 de Abril de 2013 y aceptado para ser publicado el 12 de Julio de 2013.

efecto simultáneo de los alimentos ingeridos. Los factores potenciadores aumentan las biodisponibilidad del hierro y pueden encontrarse principalmente en frutas y verduras (3). Desde el año 1987 se han diseñado diferentes intervenciones nutricionales para la prevención y control de la anemia por deficiencia de hierro en preescolares, enmarcadas en el Plan Nacional para la Prevención y Control de la Deficiencia de Hierro y la Anemia en Cuba (PN) (4), siendo el diseño de programas de intervención con alimentos fortificados con hierro, una de sus principales acciones costo-efectivas evaluada 
internacionalmente $(2,5)$

Desde el año 2002 las cinco provincias orientales son beneficiarias del Proyecto del gobierno cubano 10032 con el apoyo financiero 10032 del Programa Mundial de Alimentos (PMA) (6). Además, el gobierno cubano ha beneficiado a grupos priorizados con intervenciones nacionales de distribución subsidiada de alimentos fortificados mediante la red de comercio minorista, como son los puré de frutas (compotas) fortificados con hierro y vitamina $C$ a los niños de 6 meses a 3 años y a partir del 2005 la entrega de leche fortificada con hierro y zinc a los lactantes de todo el país (1).

En diversos países de América Latina la evaluación de los procedimientos para medir resultados, procesos e impactos son escasos, y se ha reportado que apenas un 10\% de los programas alimentarios tienen adecuados procesos de evaluación (7). Además, la evaluación de los programas de ayuda alimentaria se centran en el impacto de indicadores antropométricos, y muy escasamente realizan el análisis de estado de micronutrientes después de la intervención (7)

El objetivo de este estudio fue estimar el consumo de alimentos ricos en hierro y potenciadores de su absorción en niños de 6 meses hasta 5 años de edad, residentes en las cinco provincias orientales de Cuba en tres años dentro de una década y estimar su asociación con la anemia.

\section{SUJETOS Y MÉTODOS}

Se diseñó un estudio descriptivo de corte transversal que incluyó la evaluación intermedia y final del proyecto del gobierno cubano asistencia alimentaria 10032 con el apoyo financiero del Programa Mundial de Alimentos (PMA) y una evaluación del proyecto 10589 Apoyo al Plan Nacional para la Prevención y Control de la Anemia en las cinco provincias orientales de Cuba. El estudio se realizó en el primer semestre de los años 2005, 2008 y 2011 en las cinco provincias orientales de Cuba (Las Tunas, Holguín, Granma, Santiago de Cuba y Guantánamo).

El universo estuvo conformado por todos los niños de seis hasta 59 meses. La muestra se seleccionó teniendo en cuenta la zona de residencia (urbana o rural) y la asistencia o no de los niños al círculo infantil de cada una de las cinco provincias orientales.

Se planificó el muestreo el año 2005 que incluyó niños y niñas de 4 municipios de cada provincia y de acuerdo con sus diferencias en zona (urbana y rural), donde se consideró el municipio capital autorrepresentado. La muestra se calculó calculada para un nivel de significación del 5\% y una potencia del $90 \%$ para un total de niños por provincia de 470 y en la región de 2350. En cada provincia se seleccionarían 235 niños de zona urbana y 235 de zona rural. Esta estrategia de muestreo se utilizó también para el trabajo realizado en 2008 y 2011, lo que permitiría comparar los resultados entre ellos.

Para la selección muestral se diseñó un muestreo estratificado por conglomerado polietápico en los grupos poblacionales, donde la inclusión o no del niño en el Círculo Infantil, la zona de residencia y la provincia constituyeron los estratos y los municipios las unidades de primera etapa (UPE), los consultorios las unidades de segunda etapa (USE) y dentro de ellos los niños que constituyeron las unidades de análisis.

Dentro de cada provincia se seleccionaron de forma aleatoria y con probabilidades proporcionalmente a su tamaño 4 ó 5 municipios (aproximadamente el 40\% del total), donde siempre estuvo representado el municipio cabecera por tener la mayor cantidad de población.

De los municipios seleccionados se tomó aproximada- mente $20 \%$ de los consultorios del médico de la familia que los integraban y para lograr que los consultorios se encontraran dispersos en todo el municipio, se seleccionaron por muestreo sistemático en fases.

Con las condiciones del tamaño muestral y utilizando el muestreo sistemático en fases, se seleccionaron los niños de los consultorios médicos utilizando el listado actualizado de la campaña de vacunación antipoliomielítica. En las zonas de los municipios seleccionados en las que no existía Círculo Infantil, la población asignada a ésta representó caída de muestra.

Esta estrategia de muestreo se utilizó también para el trabajo realizado en 2008 y 2011, que permitió comparar los resultados entre ellos.

La evaluación de la dieta se realizó mediante una encuesta retrospectiva de frecuencia de consumo de alimentos ricos en hierro hemo (alimentos cárnicos, vísceras, productos elaborados con sangre) y no hem (huevo y leguminosas) que abarcaba el período de 6 meses anteriores al estudio. Se evaluaron los alimentos potenciadores de la absorción de hierro como vegetales y frutas con contenidos significativos de vitamina $C$.

La evaluación de la frecuencia de consumo fue considerada

- Nunca / Si no ingirió el alimento durante todo el período

- Poco frecuente / Si lo ingirió una vez mes hasta dos veces/ semana

- $\quad$ Frecuente / Si lo ingirió $\geq 3$ veces/semana

Para el caso específico del consumo de hígado, se consideró una ingestión frecuente el consumo de al menos una vez por semana por su alto contenido en hierro. análisis

Los alimentos se agruparon posteriormente para su

- Cárnicos: Hígado, vísceras, morcilla-chorizo, carnes rojas, y se incluyeron pescado y aves, considerando su aporte como hierro hemínico

- Huevo

- Vegetales: Lechuga, berro, acelga, perejil, espinaca, tomate, zanahoria, pimiento, quimbombó, habichuela.

- Frutas: Mandarina, naranja, toronja, frutabomba, guayaba, mango.

- Leguminosas: Frijoles, lentejas, chícharos.

- Alimentos fortificados: Compota, CSB (cereal consistente en mezcla de soya y maíz, fortificado con vitaminas y minerales), cereales para desayuno y pan en 2005.

- Leche evaporada fortificada sólo en 2008.

- Pan y compotas se evaluó individualmente en los años 2008 y 2011.

La evaluación del consumo de alimentos se realizó teniendo en cuenta que entre 6 y 11 meses comienza la alimentación complementaria y al año de edad ya debe haberse completado la introducción de todos los alimentos.

A cada niño se le realizó la determinación de hemoglobina $(\mathrm{Hb})$ mediante HemoCue $\beta$-Hemoglobin System (Blood hemoglobin photometer) con microcubetas con reactivo seco, utilizando baterías para el funcionamiento del equipo. La punción fue en el pulpejo del dedo del medio o anular de la mano, técnica debidamente estandarizada y aprobada para estudios de campo de evaluación de prevalencia de anemia, teniendo en cuenta que son niños preescolares en lugares remotos (8-10). El técnico que llevó a cabo la determinación fue entrenado y estandarizado antes de cada etapa del comienzo del trabajo para minimizar los errores en repetibilidad y se le entregó un instructivo para el desarrollo del trabajo.

Como puntos de corte para evaluar la anemia en preescolares se consideró $\mathrm{Hb}<110 \mathrm{~g} / \mathrm{L}$.(11) 
ANÁLISIS ESTADÍSTICO

Se preparó una base de datos de hemoglobina, edad y asistencia a círculo infantil original en Excel en cada provincia que fue enviada hacia el Instituto de Nutrición e Higiene de los Alimentos (INHA). La base de datos de las variables dietéticas se preparó en el INHA por personal entrenado y estandarizado.

Se evaluó la asociación del consumo de cada uno de los grupos de alimentos con la anemia y la asistencia o no a guarderías por chi-cuadrado.

Para la evaluación de la asociación de cada una de las variables con la anemia, se construyeron modelos de regresión logística en cada año (Wald backward method). Para el análisis de asociación se agruparon las variables dietéticas en consumo Nunca+ Poco Frecuente como riesgo teniendo en cuenta la frecuencia de los resultados. Los resultados se presentan mediante Razón de Disparidad (OR) e Intervalo de Confianza al 95\% (IC95\%).
Se utilizó el programa SPSS 13.0 para análisis estadístico de distribución de frecuencia y regresión logística.

\section{Bioética}

Se tuvieron en cuenta los acuerdos de la Asociación Médica Mundial, en la Declaración de Helsinki sobre los principios médicos para las investigaciones médicas en humanos, cumpliendo con los principios básicos establecidos(12). El proyecto fue aprobado por el Comité de Ética del Instituto de Nutrición e Higiene de los Alimentos. Se solicitó además la autorización del Grupo de Pediatría y de la Dirección Materno-Infantil del Ministerio de Salud Pública, con la que se discutieron los aspectos éticos de la investigación y se solicitó consentimiento informado a los padres o tutores de los niños. Todo niño detectado con anemia durante el estudio fue informado al Área de Salud y tratado por su médico de familia.

\section{TABLA 1}

Porcentaje de preescolares por grupo de edad que consumen frecuentemente los alimentos ricos en hierro y potenciadores de su absorción. Provincias orientales, Cuba.

\begin{tabular}{|c|c|c|c|c|c|c|}
\hline \multirow[t]{2}{*}{ Alimentos } & \multicolumn{2}{|c|}{2005} & \multicolumn{2}{|c|}{2008} & \multicolumn{2}{|c|}{2011} \\
\hline & $\begin{array}{c}6 \text { a } 11 \text { meses } \\
(\%)\end{array}$ & $\begin{array}{c}12 \text { hasta } 59 \\
\text { meses (\%) }\end{array}$ & $\begin{array}{c}6 \text { a } 11 \text { meses } \\
(\%)\end{array}$ & $\begin{array}{c}12 \text { hasta } 59 \\
\text { meses (\%) }\end{array}$ & $\begin{array}{c}6 \text { a } 11 \text { meses } \\
(\%)\end{array}$ & $\begin{array}{c}12 \text { hasta } 59 \\
\text { meses (\%) }\end{array}$ \\
\hline Hígado & 0 & 1.8 & 1.5 & 3.7 & 2.1 & 4.9 \\
\hline Carnes rojas & 29.8 & 29.1 & 40.1 & 34.5 & 45.4 & 38.2 \\
\hline Aves & 38.3 & 26.3 & 43.0 & 50.0 & 47.2 & 45.1 \\
\hline Pescado & 8.5 & 12.6 & 5.0 & 14.0 & 3.0 & 12.6 \\
\hline Huevos & 21.3 & 39.4 & 21.2 & 54.3 & 21.4 & 63.2 \\
\hline Lechuga & 2.1 & 13.4 & 0.8 & 8.5 & 2.6 & 12.8 \\
\hline Tomate & 4.3 & 46.6 & 17.2 & 49.8 & 20.1 & 56.8 \\
\hline Zanahoria & 4.3 & 1.1 & 6.4 & 4.1 & 8.9 & 3.2 \\
\hline Pimiento & 0 & 1.1 & 6.9 & 7.5 & 3.0 & 3.4 \\
\hline Quimbombó & 0 & 2.2 & 5.3 & 7.0 & 5.2 & 4.6 \\
\hline Habichuela & 2.1 & 3.3 & 5.7 & 8.4 & 6.9 & 9.1 \\
\hline Mandarina & 76.6 & 61.9 & 1.5 & 17.3 & 7.7 & 26.7 \\
\hline Naranja & 91.5 & 66.3 & 9.6 & 31.9 & 16.1 & 36.6 \\
\hline Frutabomba & 0 & 6.3 & 2.6 & 4.9 & 6.0 & 12.1 \\
\hline Guayaba & 25.5 & 32.3 & 5.3 & 25.1 & 13.5 & 27.3 \\
\hline Mango & 6.4 & 32.0 & 21.9 & 45.1 & 18.4 & 37.6 \\
\hline Frijoles & 91.5 & 85.3 & 59.6 & 72.7 & 43.0 & 58.0 \\
\hline Chícharos & 81.1 & 69.6 & 51.1 & 62.2 & 8.6 & 15.9 \\
\hline Leche evaporada fortificada & --- & --- & 78.2 & 16.4 & --- & --- \\
\hline Compota & --- & --- & 31.0 & $21.8^{* *}$ & 22.4 & $15.9^{* *}$ \\
\hline CSB o Fortachón & --- & --- & 0 & 0.6 & 9.4 & 9.1 \\
\hline $\operatorname{Pan}^{*}$ & 93.6 & 89.2 & 65.9 & 96.3 & 69.7 & 96.7 \\
\hline Cereales para desayuno & 0 & 2.3 & 1.9 & 1.7 & 7.3 & 4.5 \\
\hline
\end{tabular}




\section{RESULTADOS}

Se obtuvieron los datos de la dieta de 1285 niños el año 2005, 1967 el año 2008 y 2160 el 2011.

En la tabla 1 se exponen los porcentajes de consumo frecuente de los alimentos más significativos recogidos en la encuesta dietética.

\section{Grupo de 6 a 11 meses}

En este grupo el consumo frecuente de hígado se incrementó ligeramente a través de los años pero no alcanzó el 5\%, sin embargo se observó un incremento del consumo de carnes rojas y aves, con un detrimento en el consumo de pescado. El consumo de huevo se mantuvo estable durante todos los años.

Dentro de los vegetales se observó un incremento en el consumo de tomate, superior al de la zanahoria y la habichuela, comenzándose a introducir el pimiento y el quimbombó en la dieta de los niños. El consumo frecuente de lechuga resultó inferior al 5\% con pequeños cambios fluctuantes en su porcentaje.

Dentro de las frutas, el año 2005 se observó un alto consumo de naranja y mandarina que cayó significativamente en los años 2008 y 2011. La cuarta parte de los niños consumían guayaba el 2005 y decayó significativamente en los próximos años. El consumo de mango se incrementó del 2005 al 2011 pero no se incluyó en la dieta de más del $75 \%$ de los lactantes.

El consumo de frijoles y chícharos decreció paulatinamente en el período analizado. Teniendo en cuenta el consumo de alimentos fortificados, diseñados y ofertados para esta población (compota, CSB o Fortachón que es un cereal de maíz y soya fortificado), se encontró que el análisis en su conjunto como pan y otros alimentos fortificados en 2005 fue alta, a expensas principalmente del pan. En el año 2008 estaba implementada la entrega de leche evaporada fortificada con hierro y zinc que se ofertaba a todos los lactantes del país y se encontró que más del $75 \%$ de la población consumía este producto. El análisis de la frecuencia de consumo de los alimentos individuales a partir de 2008 mostró que el pan era consumido frecuentemente por más de las dos terceras partes de este grupo, el consumo frecuente de compotas sólo se encontró en la tercera parte de la población. El consumo frecuente de cereales para el desayuno ofertados en las tiendas recaudadoras de divisas no alcanzaba al $10 \%$ de la población.

\section{Grupo de 12 hasta 59 meses}

El consumo de carnes rojas y aves se incrementó también a través de los años, manteniéndose el consumo de pescado. En este grupo se incrementó significativamente el consumo de huevos. El consumo frecuente de hígado se incrementó ligeramente a través de los años pero no alcanzó el $5 \%$.

El tomate era el vegetal que era consumido frecuentemente por el mayor número de los niños y su consumo se incrementó durante este período. El vegetal mayormente consumido después es la lechuga no alcanzó el 15\% en ninguna de las evaluaciones. El porcentaje de niños que consumió frecuentemente el resto de los vegetales evaluados fue muy bajo, con un ligero incremento de ingestión de la habichuela.

Este grupo ingirió frecuentemente naranja, mandarina, guayaba y mango en 2005, observándose a partir de esta fecha un comportamiento similar al descrito en el grupo de 6 a 11 meses.

La leche evaporada fortificada con hierro y zinc estaba incluida en las dietas médicas de los niños intolerantes a otras leches hasta los 3 años de vida, por lo que encontramos un bajo porcentaje de niños que la consumieron el año 2008.

La ingestión de la compota en los niños hasta 3 años tuvo valores que decrecieron entre 2008 y 2011 . El pan era consumido por la mayor parte de la población infantil y se incrementó el consumo de cereales para el desayuno en este grupo, pero no alcanzó 5\% de la población estudiada.

Las vísceras, morcillas o chorizos, berro, acelga, perejil, espinaca, lentejas y la toronja fueron raramente consumidas en la población estudiada.

\section{GRÁFICO 1}

Frecuencia de consumo de los grupos de alimentos, preescolares de las provincias orientales, Cuba 2005.

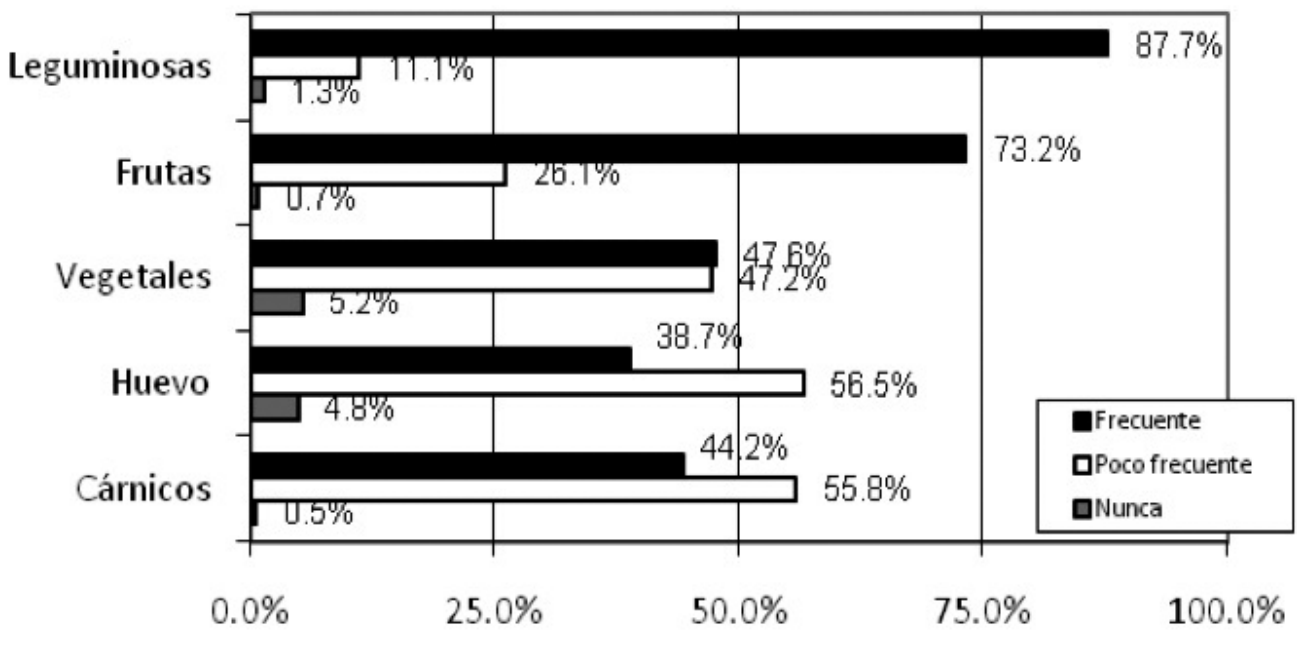


Al agrupar los alimentos para su análisis, se observó que el número de niños con consumo frecuente de alimentos cárnicos se incrementó gradualmente de $44.2 \%$ en el 2005 a $60.4 \%$ en 2011. El porcentaje de niños con ingestión frecuente de huevos también se incrementó de $38.7 \%$ en 2005 a $58.6 \%$ en 2011, así como los vegetales (47.6\% 2005 a 56.2\% en 2011). Se observó un descenso del número de niños con consumo frecuente de frutas (73.2\% en 2005 a $55.8 \%$ en 2011) y de leguminosas (87.7\% en 2005 a 57.7\% en 2011) (gráficos 1, 2,3).

El resultado del estudio de la prevalencia de anemia, disminuyó significativamente, en el grupo de 6 a 11 meses de 62.1\% en 2005 a 44.3\% en 2011 pero continúa considerándose un Problema de Salud Pública Grave para este grupo de edad. Entre el período 2005 a 2008 se observó una disminución en el grupo de 12 a 23 meses pero después se revierte y se nota un ligero ascenso, comportamiento similar se expresa

\section{GRÁFICO 2}

Frecuencia de consumo de los grupos de alimentos, preescolares de las provincias orientales, Cuba 2008.

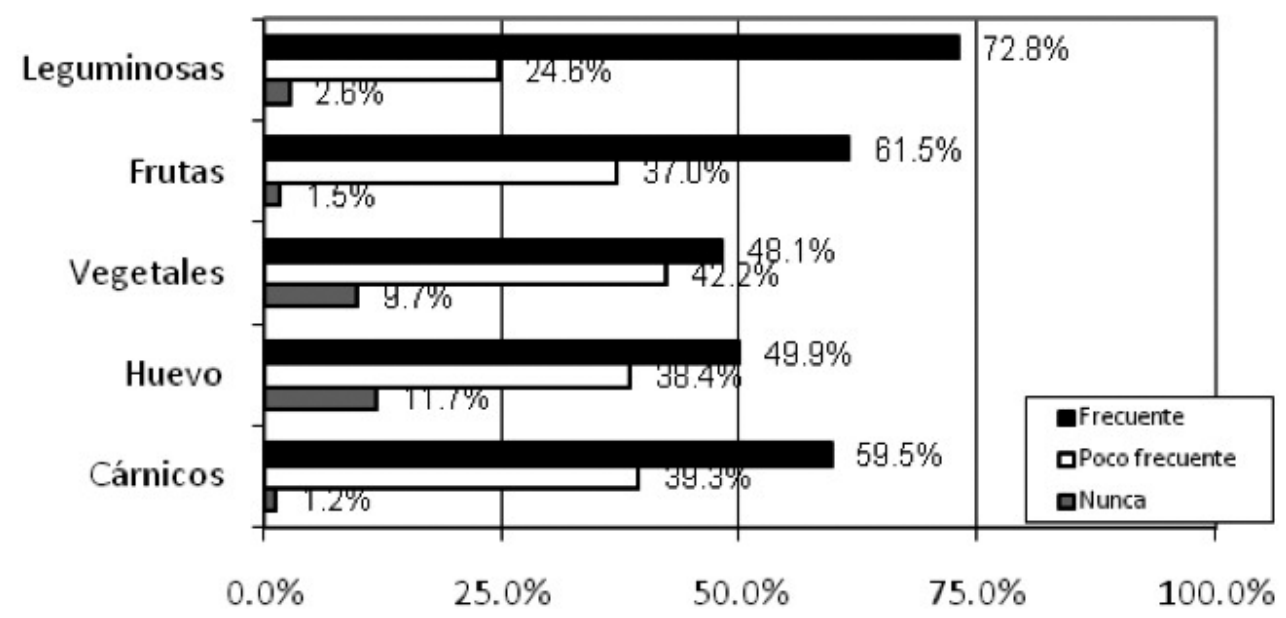

\section{GRÁFICO 3}

Frecuencia de consumo de grupos de alimentos, preescolares de las provincias orientales, Cuba 2011.

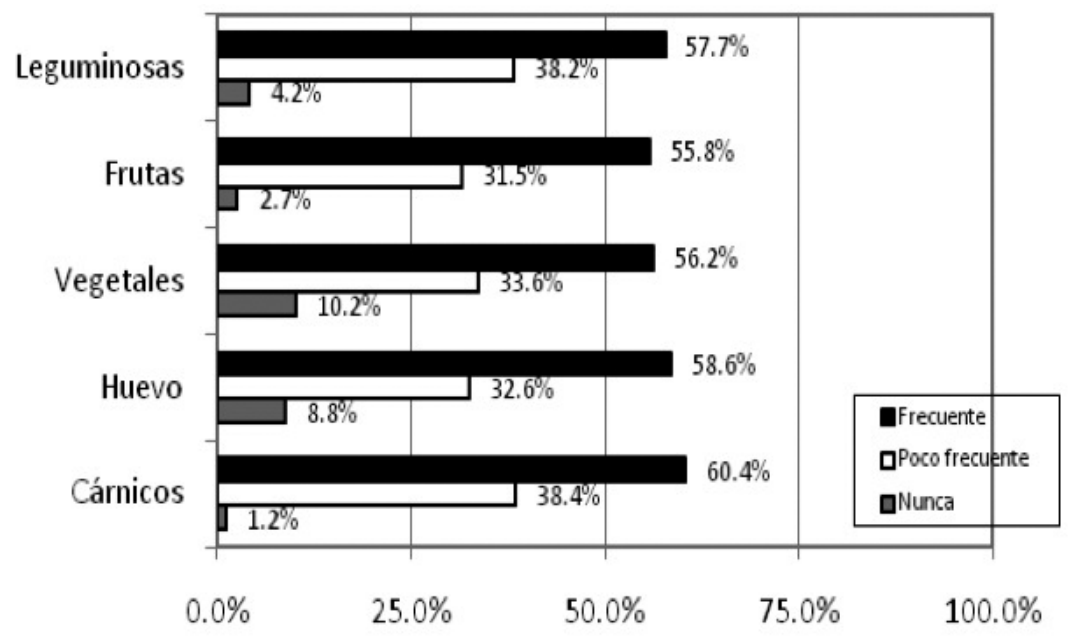


en el grupo de 24 a 59 meses (tabla 2).

El análisis de asociación de la anemia con la frecuencia de consumo por grupos de alimentos mostró asociación significativa con el consumo de alimentos cárnicos en el último año del estudio. Se encontró consistencia en la asociación significativa de la anemia con el bajo consumo de huevo, vegetales y frutas en los tres años estudio; no así con el consumo de leguminosas. El bajo consumo de pan se asoció significativamente a la anemia en los años 2008 y 2011 cuando se analizó independientemente, no así en el 2005 que se incluyó junto con la compota, CSB y cereales para desayuno.

La frecuencia de consumo de leche evaporada estuvo significativamente asociada con la anemia. Sin embargo, en el grupo de niños con anemia se registró el mayor porcentaje de niños con consumo frecuente de este alimento (tabla 3).

Los niños que asistían a guardería tenían menos riesgo de padecer anemia que los que no asistían a guardería. Este factor protector fue consistente en las tres evaluaciones realizadas (datos en proceso de publicación). El análisis de la ingestión de alimentos, evaluada de acuerdo a la asistencia a guardería, mostró que en el año 2005 sólo se encontraron diferencias significativas en la frecuencia de consumo de cárnicos y huevos. En los años 2008 y 2011 se encontró que en las guarderías un mayor número de niños consumían frecuentemente cárnicos, huevos, vegetales, frutas, leguminosas y pan, significativamente superior con respecto a los niños que no asistían a guardería. La evaluación del consumo de leche evaporada mostró que un menor número de niños la consumían en la guardería (tabla 4).

En el análisis por regresión logística de asociación de la anemia de acuerdo al grupo de edad, asistencia a guardería e ingestión frecuente de grupos de alimentos, se encontró que el niño menor de 24 meses y la no asistencia a guardería constituyen un factor de riesgo significativo en los tres años de estudio. Al evaluar el efecto de las variables dietéticas, se encontraron resultados discrepantes de acuerdo con el grupo de alimento (tabla 5).

El consumo poco frecuente y nunca de vegetales se asoció con la anemia durante los años 2005 y 2008. De manera similar ocurrió con las frutas en el año 2008.

El bajo consumo de huevo se asoció no significativamente con la anemia en los años 2005 y 2008; alcanzando significación el 2011.

El consumo poco frecuente y nunca de las leguminosas el 2005 se asoció con la anemia, sin embargo el 2008 se encontró un resultado contrario. El análisis del consumo de alimentos fortificados el 2005 mostró una asociación entre la frecuencia de consumo y la anemia que resulta paradójico. Al separar el 2008 y 2011 los alimentos que integraban este grupo, el posible efecto no apareció en las variables del modelo final.

\section{DISCUSIÓN}

Los niños menores de 2 años son el grupo poblacional de mayor riesgo de anemia por deficiencia de hierro, ya que los requerimientos de este nutriente son muy elevados y no siempre la alimentación complementaria los cubre. Esto es especialmente importante en los niños entre 6 y 12 meses y los menores de 6 meses que no reciben lactancia materna exclusiva (más del $53 \%$ del total en el país según datos de la Encuesta de Indicadores Múltiples por Conglomerados, MICS por sus siglas en inglés) (13).

La importancia de realizar intervenciones nutricionales que tengan un impacto real dirigidas a estas edades radica fundamentalmente en que la anemia en el niño pequeño resulta un importante problema de salud por sus efectos adversos en el desarrollo físico e intelectual (en especial en la capacidad de aprendizaje) son de largo alcance y no reversibles aun después de curada la anemia (14).

La información sobre el consumo de grupos de alimentos puede utilizarse para investigar patrones alimentarios, y también para saber cuáles son los que predominan en su consumo (15).

Se reconoce la importancia de evaluar los patrones de ingestión de consumo de grupos de alimentos además de un nutriente aislado para entender la relación entre la ingestión de alimentos y el riesgo de enfermedad (16).

La baja frecuencia de consumo de carnes rojas, aves y pescado puede deberse por la baja disponibilidad y acceso para la población que permita cumplir con las recomendaciones nutricionales para este grupo de edad, y por los hábitos y preferencias alimentarias del cubano (17). El bajo consumo de alimentos fuente de hierro hemínico en toda la población estudiada pudiera explicar la no asociación encontrada con la anemia ya que cuando un factor de riesgo se distribuye ampliamente en la población es muy difícil explicar su influencia en la distribución de la enfermedad (18).

La disminución brusca del consumo de cítricos, particularmente los bajos consumos encontrados de naranja y mandarina en los años 2008 y 2011, corresponde con la disminución de la producción en el país (19). También se produjo una disminución en la producción de tomate y su consumo

TABLA 2

Prevalencia (\%) e Intervalo de Confianza de la anemia en preescolares de las provincias orientales. Cuba.

\begin{tabular}{lccc}
\hline Grupos de edad & Año $2005(\mathrm{n}=2016)$ & Año $2008(\mathrm{n}=2122)$ & Año $2011(\mathrm{n}=2204)$ \\
$\%$ & $\%$ & 50.1 & 44.3 \\
6 a 11 meses & 62.1 & $(45.0-55.2)$ & $(38.3-50.3)$ \\
& $(56.1-68.7)$ & 32.7 & 37.4 \\
12 a 23 meses & 53.4 & $(28.7-36.7)$ & $(33.6-41.2)$ \\
& $(48.6-58.2)$ & 12.3 & 17.0 \\
24 hasta 59 meses & 19.8 & $(10.5-14.1)$ & $(15.0-19.0)$ \\
& $(17.7-21.9)$ & 24.0 & 26.0 \\
Total & 32.5 & $(22.2-25.8)$ & $(24.2-27.8)$
\end{tabular}


se incrementó en la población, y esto pudiera explicarse, porque dentro del grupo de los vegetales es preferido por la población cubana (17).

Es difícil evaluar en estudios epidemiológicos el efecto de los moduladores de la absorción del hierro no hemínico en los marcadores del estado de hierro (hemoglobina y ferritina sérica) debido a la exactitud del dato para la estimación de la calidad de la dieta y las interacciones entre los modificadores de su absorción. Los estudios observacionales están afectados por diversos factores de confusión como pueden ser enfermedades infecciosas o inflamatorias (20). En este estudio, se encontró una asociación de la anemia con el consumo poco frecuente y nunca de vegetales en los tres años y el consumo poco frecuente y nunca de frutas el 2008, manteniendo la asociación al ajustar el efecto de las variables en la regresión logística el 2005 y 2008.

Los adultos determinan en gran parte los hábitos alimentarios de los niños en las edades estudiadas. La encuesta de consumo, gustos y preferencias de alimentos llevada a cabo en 2001 en personas de 15 años y más muestra que la población satisface sólo el 64\% de las recomendaciones alimentarias para el hierro. El consumo adecuado de frutas y vegetales fue reportado sólo el $16.0 \%$ y $17.0 \%$, respectivamente de los encuestados y fueron los grupos de alimentos menos consumidos, en comparación con las recomendaciones (19).

Los hábitos de consumo de alimentos de una población no cambian en tan poco tiempo. Estos hallazgos correspondencia con muchos de los resultados encontrados y reflejan la necesidad de una educación alimentaria y nutricional desde la más temprana edad que contribuya a lograr una alimentación

TABLA 3

Porcentaje de preescolares según la frecuencia de consumo de los grupos de alimentos, de acuerdo a la presencia de anemia. Provincias orientales, Cuba.

\begin{tabular}{|c|c|c|c|c|c|c|c|c|c|}
\hline $\begin{array}{l}\text { Consumo por } \\
\text { grupos de } \\
\text { alimentos }\end{array}$ & Anemia & $\begin{array}{c}2005 \\
\text { No } \\
\text { Anemia }\end{array}$ & $\begin{array}{c}P \\
x^{2}\end{array}$ & Anemia & $\begin{array}{c}2008 \\
\text { No } \\
\text { Anemia }\end{array}$ & $\begin{array}{c}P \\
x^{2}\end{array}$ & Anemia & $\begin{array}{c}2011 \\
\text { No } \\
\text { Anemia }\end{array}$ & $\begin{array}{c}P \\
x^{2}\end{array}$ \\
\hline \multicolumn{10}{|l|}{ Cárnicos } \\
\hline Frecuente (\%) & 41.0 & 45.6 & \multirow[t]{3}{*}{0.191} & 56.0 & 60.5 & \multirow[t]{3}{*}{0.137} & 63.1 & 59.4 & \multirow[t]{3}{*}{0.021} \\
\hline Poco frecuente (\%) & 58.2 & 54.1 & & 42.3 & 38.4 & & 34.9 & 39.7 & \\
\hline Nunca (\%) & 0.8 & 0.3 & & 1.8 & 1.1 & & 2.0 & 0.9 & \\
\hline \multicolumn{10}{|l|}{ Huevos } \\
\hline Frecuente (\%) & 32.6 & 41.3 & \multirow[t]{3}{*}{0.002} & 39.1 & 53.0 & \multirow[t]{3}{*}{0.000} & 48.1 & 62.3 & \multirow[t]{3}{*}{0.000} \\
\hline Poco frecuente (\%) & 60.2 & 54.9 & & 42.1 & 37.3 & & 38.9 & 30.3 & \\
\hline Nunca (\%) & 7.2 & 3.8 & & 18.8 & 9.7 & & 13.0 & 7.4 & \\
\hline \multicolumn{10}{|l|}{ Leguminosas } \\
\hline Frecuente (\%) & 84.3 & 89.1 & \multirow[t]{3}{*}{0.050} & 74.4 & 72.3 & \multirow[t]{3}{*}{0.622} & 53.5 & 59.1 & \multirow[t]{3}{*}{0.000} \\
\hline Poco frecuente (\%) & 13.9 & 9.9 & & 22.9 & 25.1 & & 39.5 & 37.7 & \\
\hline Nunca (\%) & 1.8 & 1.0 & & 2.7 & 2.6 & & 7.0 & 3.2 & \\
\hline \multicolumn{10}{|l|}{ Vegetales } \\
\hline Frecuente (\%) & 40.7 & 50.5 & \multirow[t]{3}{*}{0.006} & 36.1 & 51.6 & \multirow[t]{3}{*}{0.000} & 50.6 & 58.1 & \multirow[t]{3}{*}{0.000} \\
\hline Poco frecuente (\%) & 53.3 & 44.6 & & 48.3 & 40.4 & & 32.9 & 33.9 & \\
\hline Nunca (\%) & 6.0 & 4.9 & & 15.5 & 8.0 & & 16.5 & 8.0 & \\
\hline \multicolumn{10}{|l|}{ Frutas } \\
\hline Frecuente (\%) & 71.3 & 74.0 & \multirow[t]{3}{*}{0.042} & 51.9 & 64.4 & \multirow[t]{3}{*}{0.000} & 63.7 & 95.4 & \multirow[t]{3}{*}{0.000} \\
\hline Poco frecuente (\%) & 27.2 & 25.6 & & 45.9 & 34.4 & & 30.8 & 3.2 & \\
\hline Nunca (\%) & 1.6 & 0.3 & & 2.2 & 1.3 & & 5.6 & 1.4 & \\
\hline \multicolumn{10}{|c|}{ Pan y otros alimentos fortificados } \\
\hline Frecuente (\%) & 90.3 & 87.9 & \multirow[t]{3}{*}{0.312} & & & & & & \\
\hline Poco frecuente (\%) & 7.9 & 10.6 & & & & & & & \\
\hline Nunca (\%) & 1.8 & 1.6 & & & & & & & \\
\hline \multicolumn{10}{|l|}{ Pan } \\
\hline Frecuente (\%) & & & & 87.4 & 93.5 & \multirow[t]{3}{*}{0.000} & 91.0 & 95.4 & \multirow[t]{3}{*}{0.001} \\
\hline Poco frecuente (\%) & & & & 4.9 & 2.9 & & 5.9 & 3.2 & \\
\hline Nunca (\%) & & & & 7.8 & 3.6 & & 3.1 & 1.4 & \\
\hline \multicolumn{10}{|l|}{ Compota } \\
\hline Frecuente (\%) & & & & 26.8 & 22.2 & 0.000 & 19.7 & 15.6 & 0.000 \\
\hline Poco frecuente (\%) & & & & 59.6 & 51.8 & & 65.4 & 61.5 & \\
\hline Nunca (\%) & & & & 13.6 & 26.2 & & 15.0 & 23.0 & \\
\hline Leche evaporada & & & & & & & & & \\
\hline Frecuente (\%) & & & & 38.6 & 22.6 & 0.0000 & & & \\
\hline Poco frecuente (\%) & & & & 7.2 & 5.0 & & & & \\
\hline Nunca (\%) & & & & 54.2 & 72.4 & & & & \\
\hline
\end{tabular}


saludable en niños, familia en general y educadores $(21,22)$.

En Cuba existen las Guías Alimentarias para niños hasta 2 años de edad (23) y para la población cubana mayor de 2 años (24), y un Manual de Apoyo de consejería en alimentación y nutrición (21) que permite realizar el trabajo de educación en alimentación y nutrición a los profesionales de la salud.

La disminución observada el 2008 de la prevalencia de anemia respecto al 2005 pudiera estar asociada a las acciones desarrolladas en las provincias orientales dentro del Programa Materno-Infantil, como la atención prenatal a la embarazada dirigida a la disminución del bajo peso al nacer y la anemia durante el embarazo, actividades educativas para el incremento de la lactancia materna y la vigilancia de la anemia indicada en la puericultura que permite la toma de acciones en los niños diagnosticados con anemia $(25,26)$.
Por otra parte, la puesta en marcha de las diferentes estrategias de fortificación de alimentos como la fortificación de la leche con hierro y zinc (10 mg de hierro y $5 \mathrm{mg}$ de zinc por cada litro de leche) (27) y la compota fortificada con hierro y vitamina $C$ no mostraron un efecto en la reducción de la anemia, evidenciándose que un factor de riesgo de gran importancia en el desarrollo de la anemia es la edad del niño.

A pesar de que la absorción del hierro no hemínico es baja, los alimentos portadores de este tipo de hierro (huevo y leguminosas) son un una fuente importante de este mineral en la dieta de los cubanos, por la frecuencia y cantidad de consumo (3). Esto explica la asociación encontrada en los tres años de estudio entre el bajo consumo de huevo y desarrollo de la anemia, siendo el único factor dietético encontrado con asociación significativa en la regresión logística en el 2011.

\section{TABLA 4}

Porcentaje de preescolares según la frecuencia de consumo de los grupos de alimentos, de acuerdo a la asistencia a guardería. Provincias orientales, Cuba.

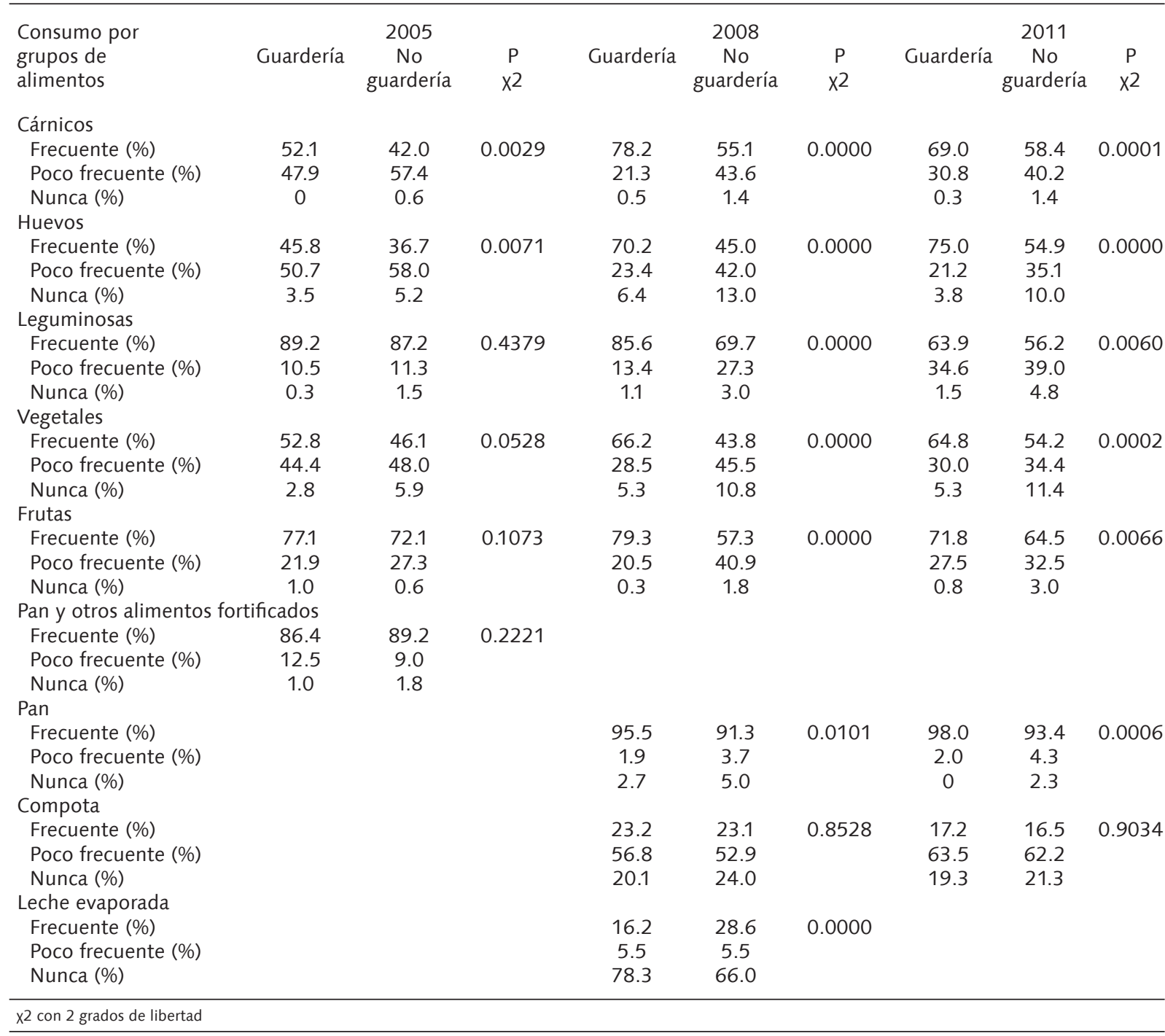


La progresiva disminución del consumo de leguminosas podría obedecer, a la baja producción nacional de granos y al acceso a los mismos (19). El análisis de la asociación del consumo de leguminosas con la anemia mostró resultados discordantes que pudieran explicarse por la disminución brusca en el consumo frecuente y el incremento de consumo de otros alimentos fuentes de hierro o favorecedores de su absorción el 2008 y 2011. Este aspecto necesitaría estudios cuantitativos posteriores que permitan evaluar con el peso de este alimento en el análisis.

Como limitación en este estudio se encontró que la evaluación del 2005 se realizó teniendo en cuenta los alimentos fortificados en un sólo grupo, obteniéndose resultados contrarios al efecto esperado en el análisis de regresión logística. Esto pudiera explicarse porque el niño que tiene un consumo frecuente de pan reduce el consumo de otros alimentos ricos en hierro y potenciadores de su absorción, sin cubrir los requerimientos de hierro. En Chile la baja prevalencia de anemia encontrada en la población de niños y mujeres en edad fértil se explica principalmente por el efecto de la fortificación de la harina de panificación con hierro (28). Al evaluar el peso de los alimentos individuales en la regresión logística el 2008 y 2011, desaparece el efecto de estas variables en el desarrollo de la anemia.

Los porcentajes de quienes consumen grupos de alimentos ricos en micronutrientes se pueden utilizar como medidas puntuales, para realizar un seguimiento continuado o para evaluar cambios en la dieta antes y después de una intervención (15).

Estudios en América Latina han evaluado el consumo de alimentos por recordatorio de 24 horas y han encontrado ingestas inadecuadas de hierro en la dieta, asociadas o no,

TABLA 5

Resultados del último paso de la regresión logística (backward method) para la asociación de la anemia en preescolares con variables dietéticas, grupos de edad y asistencia a guardería por año de estudio. Provincias orientales, Cuba.

\begin{tabular}{|c|c|c|c|c|c|c|c|c|c|}
\hline \multirow[t]{2}{*}{ Variables } & \multicolumn{3}{|c|}{$2005 n=1266$} & \multicolumn{2}{|c|}{$2008 n=1564$} & \multicolumn{4}{|l|}{$2011 n=2055$} \\
\hline & $\mathrm{p}$ & OR & IC95\% & $\mathrm{p}$ & OR & IC95\% & p & OR & IC95\% \\
\hline \multicolumn{10}{|c|}{ Edad (meses) } \\
\hline$<24 / 324$ & 0.000 & 5.861 & $4.353-7.890$ & 0.000 & 3.724 & $2.865-4.840$ & 0.000 & 2.712 & $2.191-3.358$ \\
\hline \multicolumn{10}{|c|}{ Asistencia a guardería } \\
\hline $\mathrm{No} / \mathrm{Si}$ & 0.000 & 2.327 & $1.608-3.67$ & 0.008 & 1.754 & $1.159-2.656$ & 0.000 & 2.642 & $1.863-3.748$ \\
\hline \multicolumn{10}{|c|}{ Frecuencia consumo carnes } \\
\hline \multicolumn{10}{|c|}{ Nunca+Poco Frecuente/Frecuente } \\
\hline & 0.053 & 1.316 & $0.997-1.736$ & & & & & & \\
\hline \multicolumn{10}{|c|}{ Frecuencia consumo vegetales } \\
\hline \multicolumn{10}{|c|}{ Nunca+Poco Frecuente/Frecuente } \\
\hline & 0.049 & 1.315 & $1.001-1.729$ & 0.000 & 1.739 & $1.276-2.372$ & & & \\
\hline \multicolumn{10}{|c|}{ Frecuencia consumo Frutas } \\
\hline & & & & 0.024 & 1.431 & $1.048-1.956$ & & & \\
\hline \multicolumn{10}{|c|}{ Frecuencia consumo huevos } \\
\hline \multicolumn{10}{|c|}{ Nunca+Poco Frecuente/Frecuente } \\
\hline & 0.427 & $1.132^{*}$ & $0.827-1.550$ & 0.052 & 1.352 & $0.988-1.833$ & 0.032 & 1.264 & $1.021-1.566$ \\
\hline \multicolumn{10}{|c|}{ Frecuencia consumo leguminosas } \\
\hline & 0.001 & 1.941 & $1.295-2.909$ & 0.000 & 0.500 & $0.358-0.698$ & & & \\
\hline
\end{tabular}

Frecuencia consumo pan y otros alimentos fortificados

Nunca+Poco Frecuente/Frecuente

$$
0.006 \quad 0.518 \quad 0.325-0.825
$$

Valor $\mathrm{p}$ de Wald del modelo

Variables incluidas en el modelo inicial. Backward method (Wald) Edad $<24 / 324$ meses

Asistencia a guardería No/Si

Frecuencia consumo de cárnicos Nunca+Poco Frecuente/Frecuente

Frecuencia consumo huevos Nunca+Poco Frecuente/Frecuente *Eliminado en el último paso de análisis.

Frecuencia consumo leguminosas Nunca+Poco Frecuente/Frecuente

Frecuencia consumo vegetales Nunca+Poco Frecuente/Frecuente

Frecuencia consumo frutas Nunca+Poco Frecuente/Frecuente

Frecuencia consumo pan y otros alimentos fortificados en 2005 Nunca+Poco Frecuente/Frecuente

Frecuencia consumo pan en 2008 y 2011 Nunca+Poco Frecuente/Frecuente

Frecuencia consumo compota 2008 y 2011 Nunca+Poco Frecuente/Frecuente

Frecuencia consumo leche evaporada 2008 Nunca+Poco Frecuente/Frecuente 
con bajos niveles de hemoglobina (29-32).

Uno de los mayores retos de la epidemiología nutricional es medir la dieta en un número grande de personas con suficiente precisión y a la vez, con un costo relativamente bajo, para relacionar la ingestión de alimentos y nutrientes con las enfermedades (33). El análisis de la evaluación dietética realizada y su relación con la anemia ha resultado difícil de explicar, al no poder profundizar en el aporte específico de micronutrientes de los alimentos.

La información detallada sobre acceso de alimentos en el hogar o el consumo alimentario individual requiere de tiempo, recursos y una alta capacidad técnica para recopilación de los datos y su análisis. La evaluación de la diversidad alimentaria es una medida cualitativa de consumo de alimentos y refleja el acceso de los hogares a una variedad de alimentos, e indirectamente, a la adecuación de nutrientes de la dieta individual. Un incremento en la diversidad alimentaria se correlaciona con una mayor adecuación nutricional en la dieta (15).

La sensibilidad de la determinación de hemoglobina como indicador de deficiencia de hierro es baja, a menudo se solapan los valores entre los individuos deficientes de hierro y los individuos sanos. Si bien se acepta que en poblaciones con alta prevalencia de anemia, la causa fundamental es la deficiencia de hierro, es fundamental en estos lugares estimarla mediante otras pruebas bioquímicas (1). Otras deficiencias nutricionales pueden ser responsables, además, de mantener las elevadas prevalencias de anemia encontradas como las deficiencias de cobalamina, ácido fólico, vitamina A, riboflavina, vitamina $C$, cobre y zinc; y no nutricionales como infección e inflamación, (34-36).

\section{CONCLUSIONES Y RECOMENDACIONES}

El bajo consumo de huevos, vegetales y frutas constituye un factor de riesgo alimentario de desarrollo de la anemia en los niños preescolares. Los niños que asisten a guardería consumen alimentos ricos en hierro y potenciadores de su absorción con mayor frecuencia que los que no asisten, por lo que la asistencia a guardería constituye un factor protector para la anemia en esta población. Los niños menores de 24 meses se mantienen como el grupo de mayor vulnerabilidad para el desarrollo de anemia. Estos resultados sugieren la necesidad de reforzar las acciones de educación alimentaria con el objetivo de lograr en la alimentación de los preescolares una correcta combinación de alimentos para incrementar la ingestión y biodisponibilidad del hierro.

\section{RESUMEN}

La anemia en niños menores de 5 años constituye el principal problema nutricional en Cuba. El objetivo de este estudio fue estimar el consumo de alimentos ricos en hierro y potenciadores de su absorción en niños residentes en las cinco provincias orientales de Cuba y la asociación con la anemia en los años 2005, 2008 y 2011. La evaluación se realizó mediante una encuesta de frecuencia de consumo de alimentos en el período de 6 meses anterior a la fecha del estudio y determinación de hemoglobina mediante HemoCue. El consumo frecuente entre 2005 a 2011 incrementó en cárnicos $(44.2 \%$ a $60.4 \%$ ), huevos (38.7\% a $58.6 \%$ ) y vegetales (47.6\% a $56.2 \%$ ); con un descenso en frutas $(73.2 \%$ a $55.8 \%$ ) y leguminosas (87.7\% a 57.7\%). La prevalencia de anemia disminuyó en niños de 6 a 11 meses de 2005 a 2011 (62.1\% a 44.3\%). Se observó disminución de la anemia en el grupo de 12 a 23 meses en 2008 que revierte ligeramente el 2011, de manera similar ocurrió en el grupo de 24 a 59 meses. Se encontró asociación de la anemia con el bajo consumo de huevo, vegetales y frutas; no con el consumo de leguminosas. Los niños que asisten a guardería consumían alimentos ricos en hierro y potenciadores de su absorción con mayor frecuencia que los que no asistían, por lo que la asistencia a guardería constituye un factor protector para la anemia en esta población. El grupo de niños menores de 24 meses se mantiene como el grupo de mayor vulnerabilidad para la anemia.

Palabras clave: alimentos ricos en hierro; anemia; preescolares; guardería.

Agradecimientos: Agradecemos a los nutriólogos, dietistas y personal de los Centros Provinciales de Higiene y Epidemiología en la participación para la toma de muestra y encuestas realizadas. En Las Tunas: Irelis Tamayo, Osmani Mendez; Holguín: Joan Araluce, Clara Rosa Brau y Jimmy Campos; Santiago de Cuba: Jorge Ruíz, Guantánamo: Esneiva Venereo. A la Dra. Isabel Barroso Utra, Lic. José Reboso Pérez y Dr. René Guillermo García Roche por el diseño estadístico del proyecto. Al Dr. Enrique Molina Esquivel por su asesoramiento en el análisis estadístico. A la oficina del Programa Mundial de Alimentos en Cuba por su apoyo en la logística y organización del trabajo. A las técnicas Caridad Arocha Oriol y Dania Herrera Javier por el apoyo sostenido, recepción de encuestas y confección de bases de datos.

\section{BIBLIOGRAFÍA}

1. Pita Rodríguez G, Jiménez Acosta S. La anemia por deficiencia de hierro en la población infantil de Cuba. Brechas por cerrar. Revista Cubana de Hematología, Inmunología y Hemoterapia. 2011;27(2):179-95.

2. WHO/CDC., editor. Worldwide prevalence of anaemia 1993-2005: WHO global database on anaemia. Geneva: WHO Press; 2008.

3. Jiménez S, Pita G, Padrón M. La anemia por deficiencia de hierro. Aspectos generales para su prevención y control. Manual de capacitación para el equipo de salud. In: Instituto de Nutrición e Higiene de los Alimentos, MINSAP, PMA, editor. La Habana 2009.

4. Padrón M. Plan integral para la prevención y el control de la anemia por deficiencia de hierro en Cuba.2009 [cited 2010 Mar 10]: Available from: www.inha.sld.cu/Documentos/ plan\%20anemia.doc

5. Horton S. The economics of nutritional intervention. In: Semba R, Bloem M, editors. Nutrition and Health in Developing Countries. 2nd ed. New Jersey: Humana Press; 2008. p. 859-71.

6. Reboso J, Jiménez S, Monterrey P, Macías C, Pita G, Selva $L$, et al. Diagnóstico de la anemia por deficiencia de hierro en niños de 6 a 24 meses y de 6 a 12 años de edad de las provincias orientales de Cuba. Rev Esp Nutr Comunitaria. 2005;11(2):60-8.

7. Galván M, Amigo H. Programas destinados a disminuir la desnutrición crónica. Una revisión en América Latina. Arch Latinoam Nutr. 2007;57(4):316-26.

8. Romero Ruiz A, Naviera Abeigón E, Gómez Alba J, Ramírez $G$, Muñoz M. Utilidad de la medición de hemoglobina con el HemoCue B Hemoglobin en el manejo de la anemia. Revisión de nuestra experiencia en distintos contextos. Anemia. 2009;2(2):21-30.

9. Neufeld L, García-Guerra A, Sánchez-Francia D, NewtonSánchez O, Ramírez-Villalobo M, Rivera-Dommarco J. Hemoglobin measured by Hemocue and a reference method in venous and capillary blood: A validation study. Salud 
Pública de México [serial on the Internet]. 2002; 44(3): Available from: http://hinari-gw.who.int/whalecomwww. scielosp.org/whalecom0/pdf/spm/v44n3/a05v44n3.pdf.

10. de A Paiva A, Rondó P, de B Silva S, Latorre M. Comparison between the HemoCue and an automated counter for measuring hemoglobin. Rev Saude Publica. 2004;38(4):585-7.

11. WHO, editor. Iron deficiency anemia. Assesment, Prevention and Control. A guide for programme managers: WHO/ NHD/01.3; 2001.

12. Declaración de Helsinki de la Asociación Médica Mundial. Principios éticos para las investigaciones médicas en seres humanos, 52 a Asamblea General, Edimburgo, Escocia, octubre 2000. Nota de clarificación del párrafo 30, agregada por la Asamblea General de la AMM, Tokio 2004. [cited 2005 Apr 30]. Available from: http://www.saber.ula.vel bitstream/123456789/16099/1/declaracion_helsinski.pdf.

13. Dirección Nacional de Estadística, Ministerio de Salud Pública, editors. Encuesta de Indicadores Múltiples por Conglomerado2012.

14. Yadav D, Chandra J. Iron Deficiency: Beyond Anemia. Indian J Pediatr 2011;78:65-72.

15. Kennedy G, Ballard T, Dop MC, editors. Guía para medir la diversidad alimentaria a nivel individual y del hogar. Roma: FAO; 2013.

16. Salvo D, Frediani JK, Ziegler TR, Cole CR. Food group intake patterns and nutrient intake vary across low-income Hispanic and African American preschool children in Atlanta: a cross sectional study. Nutrition Journal [serial on the Internet]. 2012 [cited 2012 Dec 27]; 11: Available from: http://www.ncbi.n/m.nih.gov/pmc/articles/ PMC3487737/pdf/1475-2891-11-62.pdf.

17. Porrata C. Consumo y Preferencias alimentarias de la población cubana con 15 y más años de edad. . Rev Cub Aliment Nutr 2009;19(1):87-105.

18. Rose $G$. Individuos enfermos y poblaciones enfermas. OPS Boletín Epidemiológico 1985;6(3):1-8

19. Oficina Nacional de Estadísticas de la República de Cuba. Producción agrícola por cultivos seleccionados de la agricultura no cañera.Anuario Estadístico de Cuba 2010. 2011 [cited 2013 April 2]; Available from: http://www.one.cu/ aec2010/esp/09_tabla_cuadro.htm.

20. Geissler C, Singh M. Iron, Meat and Health. Nutrients 2011;3:283-316.

21. Jiménez Acosta $S$, Rodríguez Suárez A, Pita Rodríguez $G$, Zayas Torrientes G, Díaz Sánchez ME, Castanedo Valdés $R$, et al. Consejería en alimentación y nutrición. Manual de Apoyo. In: INHA, OPS, editors. La Habana: Molinos Trade S.A.; 2011. p. 128.

22. Pita Rodríguez $G$, Basabe Tuero B, Jiménez Acosta S, Mercader Camejo O. La anemia. Prevención por medio de la alimentación. Manual para población 2007 [cited 2010 Jan 15]. Available from: www.sld.cu/galerias/pdf/sitios/ hematologia/anemia_para_poblacion_aps_2009.pdf.

23. Jiménez Acosta S, Pineda Pérez S, Sánchez Ramos R, Rodríguez Suárez A, Domínguez Ayllón Y. Guías Alimentarias para niñas y niños cubanos hasta 2 años de edad. Documento técnico para los equipos de Salud. In: INHA, PAMI/ MINSAP, editors. La Habana 2009. p. 80.

24. Porrata C, Castro D, Rodríguez L, Martín I, Sánchez $R$, Gámez Al, et al. Guías alimentarias para la población cubana mayor de dos años de edad. In: INHA, editor. La Habana2009. p. 61.

25. Experiencias exitosas. Programa Nacional Materno Infantil, Programa de suplementación con «Prenatal» en la Atención Primaria de Salud y Hogares Maternos 2012. [cited $2013 \mathrm{Jul}$ 4]Available from: http://cuba.nutrinet.org/cuba/ experiencias-exitosas

26. Grupo Nacional de Puericultura Departamento Materno Infantil. Ministerio de Salud pública. Consulta de puericultura 2011. [cited $2013 \mathrm{Jul}$ 4]. Available from: http:// files.sld.cu/puericultura/files/2012/02/libro-consulta-depuericultura-20111.pdf

27. Pita Rodríguez G, Basabe Tuero B, Díaz Sánchez ME, Mercader Camejo O, Reboso Pérez J, Carrillo Selles M, et al. Progreso en la reducción de la anemia en niños y niñas de un año de edad en La Habana entre los años 2005 y 2007. Nutr Clín Diet Hosp. 2012;32(1):13-25.

28. Olivares M, Pizarro F, Hertrahpf E, Walter T, Arredondo M, Letelier A. Fortificación de alimentos con hierro en Chile. Rev Chil Nutr. 2000;27(3):340-4.

29. Batrouni L, Piran MF, Eandi M, Dasbul G, Toledo S. Parámetros bioquimicos y de ingesta de hierro, en niños de 12 a 24 meses de edad de Córdoba, Argentina. Rev Chil Nutr [serial on the Internet]. 2004 [cited 2013 March 25]; 31(3): Available from: <http://www. scielo.cl/scielo.php?script=sci_arttext\&pid=S071775182004000300008\&/ng=es\&nrm=iso >.

30. Galván G M, Atalah S E. Variables asociadas a la calidad de la dieta en preescolares de Hidalgo, México. Rev Chil Nutr. 2008;35(4):413-20.

31. del Real SI, Sánchez-Jaeger A, Barón MA, Díaz N, Solano L, Velásquez $E$, et al. Estado nutricional en niños preescolares que asisten a un jardín de infancia público en Valencia, Venezuela. Arch Latinoam Nutr. 2007;57(3):248-54.

32. Bastardo G, Angarita C, Quintero Y, Rojas L, Rodríguez LC, Marquez JL. Consumo de hierro y otros nutrientes en la dieta de preescolares residentes en apartaderos, Escagüey y Pueblo Llano (Mérida, Venezuela). MedULA 2008;17(1):41-6.

33. Parra Cabrera S, Romieu I, Hernández Ávila M, Mardrigal Fritsh $\mathrm{H}$. Uso y limitaciones de los métodos de encuesta dietética Madrigal Fritsh H, Martínez Salgado H, editors. Cuernavaca, Morelos1996.

34. Lutter C. Iron deficiency in young children in low-income countries and new approaches for its prevention. J Nutr. 2008;138(12):2523-8.

35. Lynch $S$. Why nutritional iron deficiency persists as a worldwide problem. J Nutr. 2011 1;141(4):763S-8S.

36. Thurnham D. Monitoring Anemia-Control Program. Sight and Life Magazine. 2009:59-66 